\title{
PREDIÇÃO DE PROPRIEDADES, EQUILÍBRIO DE FASES E EQUILÍBRIO QUÍMICO PARA SUBSTÂNCIAS ENVOLVIDAS NA REAÇÃO DE TRANSESTERIFICAÇÃO
}

\author{
S. ARVELOS ${ }^{1}$, E. O. WATABABE ${ }^{1}$, C. E. HORI ${ }^{1}$ e L. L. ROMANIELO ${ }^{1}$ \\ ${ }^{1}$ Universidade Federal de Uberlândia, Faculdade de Engenharia Química \\ E-mail para contato: lucienne@ufu.br
}

\begin{abstract}
RESUMO - Por diferentes métodos de contribuição de grupos e correlações, dados termodinâmicos (parâmetros críticos, fator acêntrico, capacidade calorífica, entalpia e energia livre de Gibbs de formação) foram estimados e comparados aos dados disponíveis na literatura para tri, di e monoglicerídeos de ácidos graxos (láurico, mirístico, esteárico, palmítico, oleico, linoleico e linolênico), metil e etil ésteres e glicerol que são as principais substâncias envolvidas nas reações de transesterificação (RT) de óleos vegetais. Para predizer as condições reacionais que determinam o estado supercrítico da mistura inicial, diagramas para várias razões molares de mistura (óleo+álcool) foram modelados. A partir destes resultados, foram selecionados os métodos e correlações para se predizer o equilíbrio químico da RT com alcoóis supercríticos. Os resultados mostraram conversões totais dos triglicerídeos em todas as condições analisadas.
\end{abstract}

\section{INTRODUÇÃO}

O biodiesel é uma mistura de ésteres de ácidos graxos, derivados de óleos vegetais ou gorduras animais que são compostos, em média, de 97\% de triglicerídeos (TG). A reação de transesterificação (RT) realizada de forma catalítica é principal forma de obtenção de ésteres a partir destas moléculas. A representação da reação global se dá como na Figura 1 , na qual $R_{i}$, são radicais alquílicos; quando estes radicais são iguais denomina-se o TG como TG simples e estes podem ser de cadeia saturada ou não. Na RT de um TG simples a reação se processa em três etapas, na qual em cada uma destas um radical alquílico é retirado da molécula TG, tendo por intermediários então diglicerídeo e monoglicerídeo. Os alcoóis mais utilizados na RT são metanol e etanol (Anikeev et al., 2012).
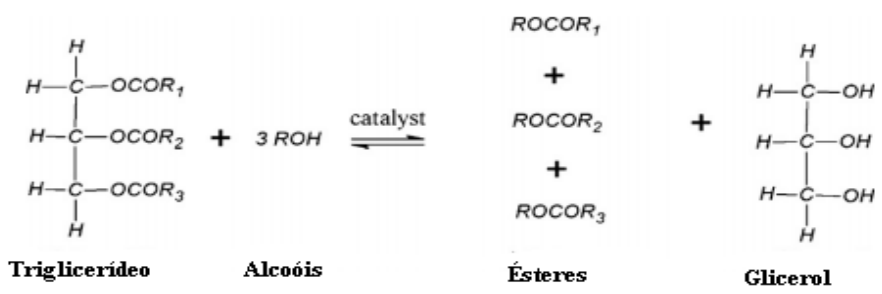

Figura 1 - Reação global de transesterificação de triglicerídeos. 
Nos últimos anos, a produção deste biocombustível por via não catalítica usando um álcool em condições supercríticas tem sido estudada devido a vantagens já bem descritas na literatura. Conversões de TG's em torno de $87-99 \%$ e rendimentos em tornos de $90-95 \%$ têm sido relatados em diversos trabalhos, sendo que as condições operacionais abrangem pressões de 100300 bar e temperaturas de 560-700 K (Anistecu et al., 2008). Contudo faz-se necessária a investigação de condições mais amenas de temperatura e pressão com possibilidade de altas conversões como as observadas e a análise do equilíbrio químico da reação global se mostra uma alternativa para esta avaliação.

O objetivo deste trabalho foi predizer as condições em que a mistura reacional inicial (óleo+álcool) de transesterificação se torna supercrítica, já pois segundo a literatura (Stepanov et $a l ., 2011)$ a reação se processa em uma única fase supercrítica quando é garantida a condição de uma única fase para os reagentes, garantindo uma reação rápida e altos rendimentos. Além disso, objetiva também calcular o rendimento em ésteres no equilíbrio químico com álcool supercrítico (Smith et al., 2000). Este trabalho também tem por meta avaliar métodos de contribuição de grupos e correlações para estimação de propriedades das substâncias puras envolvidas nos cálculos efetuados (parâmetros críticos - temperatura crítica $\left(T_{c}\right)$, pressão crítica $\left(P_{c}\right)$, volume crítico $\left(V_{c}\right)$, fator acêntrico $(\omega)$, capacidade calorífica do gás ideal $\left(C_{p}^{i g}\right)$, entalpia $\left(\Delta H_{f}^{0}(i g)\right)$ e energia livre de Gibbs de formação $\left(\Delta G_{f}^{0}(i g)\right.$. De posse destes cálculos, pode-se inferir sobre o processo de forma teórica e sugerir condições para investigação prática.

\section{METODOLOGIA}

A Tabela 1 mostra as propriedades estimadas neste trabalho e seus respectivos métodos de estimação. A formulação destes métodos pode ser obtida em Kesler e Lee (1976), Han e Peng (1993), Rihani e Doraiswamy (1965), Poling et al. (2001) e Marrero e Gani (2001). $T_{b}$ (T normal de ebulição) foi incluída nos cálculos porque dela advém a estimação de $T_{c}$ em alguns métodos, $\omega$ depende de $V_{c}, C_{p}{ }^{i g}$ depende de $C_{p}^{\text {liq }}$ (capacidade calorífica de líquidos), $\Delta H_{f(i g)}^{0}$ depende da de vaporização $\left(\Delta H^{v a p}\right)$

Tabela 1 - Propriedades estimadas e métodos de estimação utilizados

\begin{tabular}{ll}
\hline Propriedade & Método \\
\hline$T_{b}$ & Joback e Reid - MJR, Constantinou e Gani - MCG, Marrero-Marejon e Pardillo- \\
& Fontdevila - MMP, Marrero-Gani - MMG \\
$T_{c}, P_{c}$ e $V_{c}$ & Ambrose - MA, MJR, MCG e MMG \\
$\omega$ & Pitzer - RP, Ambrose e Walton - RAW, Edmister - RE, - RKL e Han e Peng- RHP \\
$C_{p}^{i g}$ & MJR, Rihani e Doraiswamy - MRD \\
$C_{p}{ }^{l i q}$ & Bondi \\
$\Delta H^{\text {vap }}$ & Reidel, Chen, Vetere, Watson, MCG \\
$\Delta H_{f(i g)}^{0} e \Delta G_{f(i g)}^{0}$ & MJR, MCG, MMG
\end{tabular}

As propriedades estimadas foram validadas com dados experimentais obtidos da literatura. As referências dos dados experimentais são apresentadas em conjunto com os resultados. Os desvios calculados são dados em termos da média dos desvios médios relativos percentuais (DMR). 


\section{9 a 22 de outubro de 2014 \\ Florianópolis/SC}

Para escolha do melhor método para o cálculo de $T_{c}, P_{c}$ e $\omega$ dados experimentais de pressão de saturação $\left(P^{v p}\right)$ e densidade de líquidos $\left(\rho^{l i q}\right)$ foram regredidos para a equação de Peng e Robinson - PR-EOS utilizando por função objetivo a média do DMR destas duas variáveis no software PE 2000 (Pfohl et al., 2000). Os parâmetros regredidos foram comparados com os estimados pelos métodos e contribuição de grupo e correlações listados na Tabela 1. Não se optou por utilizar os dados diretamente regredidos nos cálculos posteriores porque nem todas as substâncias de interesse têm dados de densidade e pressão de saturação disponíveis.

Para validação de $C_{p}{ }^{i g}$, como foram obtidos dados experimentais para líquidos, os métodos MJR e MRD foram acoplados à equação de Bondi (equação que prediz a $C_{p}$ liq a partir de $C_{p}{ }^{i g}$ ). Para avaliação da $\Delta H_{f}^{0}(i g)$ foi necessário estimar a entalpia de vaporização das substâncias à $298 \mathrm{~K}$, pois somente dados de entalpia de líquidos foram obtidas na literatura. Numa mesma temperatura, a entalpia de formação do gás é dada pela soma da entalpia de formação do líquido somando-se a entalpia de vaporização. $\mathrm{Na}$ avaliação dos métodos de predição da entalpia de vaporização, os métodos de Reidel, Chen e Vetere foram modificados pela equação de Watson.

Foram elaborados algoritmos de cálculo de ponto bolha e orvalho (em Maple 15) por configuração assimétrica utilizando a PR-EOS e regra de mistura clássica de van der Waals considerando os parâmetros de interação binários nulos visando elaborar curvas binodais para diversas razões molares de álcool:óleos. As propriedades dos óleos $\left(T_{c}, P_{c}\right.$ e $\left.\omega\right)$ foram calculados pela regras de mistura de Lorentz-Berthelot e demais propriedades por regras de misturas quadráticas sendo que a composição dos óleos foi obtida em Ma e Hanna (1999) vide Tabela 2.

Tabela 2 - Composição de óleos em termos de ácidos graxos

\begin{tabular}{ccccccccc}
\hline \multirow{2}{*}{ Óleo } & \multicolumn{8}{c}{ Conteúdo de ácidos graxos } \\
\cline { 2 - 8 } & Laurato & Miristato & Palmitato & Estearato & Oleato & Linoleato & Linolenato & Outros \\
\hline Milho & - & - & 11,67 & 1,87 & 25,16 & 60,60 & 0,48 & 0,22 \\
Algodão & 0,10 & 0,70 & 20,10 & 2,60 & 19,20 & 55,20 & 0,60 & 1,5 \\
Soja & - & 0,10 & 11,75 & 3,15 & 23,26 & 55,53 & 6,31 & 0,00 \\
Girassol & - & - & 6,08 & 3,26 & 16,93 & 73,73 & - & 0,00 \\
\hline
\end{tabular}

A partir das curvas binodais foram confeccionadas curvas do local geométrico do ponto crítico (curvas críticas) de misturas óleos+alcoóis de cadeia curta (metanol e etanol). De posse de todas estas informações e dos dados preditos, a composição do equilíbrio químico da RT em etapas de um TG simples foi calculada através de algoritmo implementado em Maple 15 utilizando a formulação estequiométrica.

\section{RESULTADOS E DISCUSSÃO}

\subsection{Estimação de Propriedades}

Os métodos de Gani (MCG e MMG) apresentam melhor capacidade de predizer temperaturas normais de ebulição para as substâncias estudadas com médias dos DMR de 8,03 e 8,60\%. Uma representação qualitativa dos resultados é apresentada na Figura 2 (a). 
Os dados de $T_{c}, P_{c}$ e $\omega$ regredidos com o PE utilizando a PR-EOS representam bem os dados de densidade e pressão de saturação experimentais consultados no DIPPR, sendo que a média dos DMR para a pressão de saturação foi de $0,78 \%$ e para a densidade de $2,45 \%$, desvios muito próximos das incertezas das medidas que são de 5 e $1 \%$, respectivamente. A Figura 2 (b) e (c) apresenta a correlação entre os valores regredidos e estimados via contribuição de grupos.

Para o uso do método de Pitzer (regra de Ptizer $=\mathrm{RP}$ ) para o cálculo de fator acêntrico, faz-se necessária a obtenção do volume crítico das substâncias avaliadas. Os valores estimados de $V_{c}$ foram comparados ao estimado utilizando o fator de compressibilidade crítico de 0,3074 da equação PREOS com $T_{c}$ e $P_{c}$ regredidos. Os métodos apresentaram estimações muito próximas com DMR em torno de 20-24\%. O método selecionado neste trabalho foi o método de Marrero e Gani para cálculo de $V_{c}$. A Figura 2 (c) mostra o ajuste geral dos fatores acêntricos estimados em relação dos regredidos. Pode-se notar que o método de Han e Peng (RHP) apresenta a melhor predição.

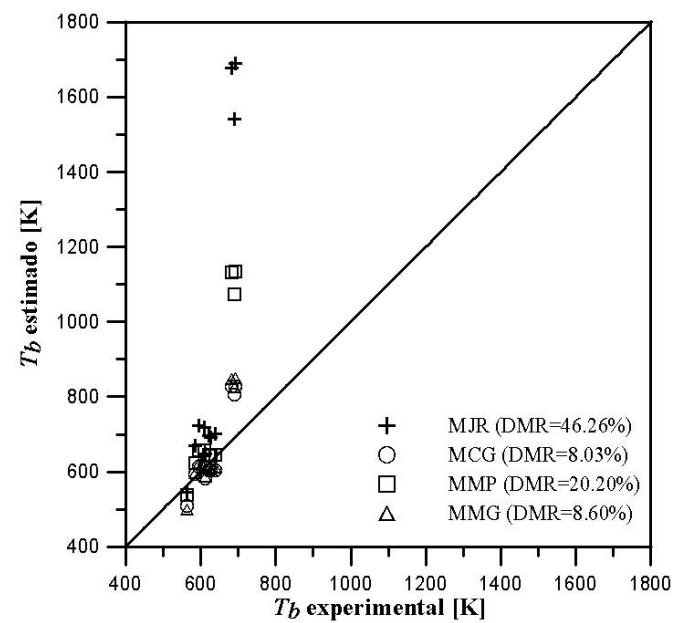

(a)

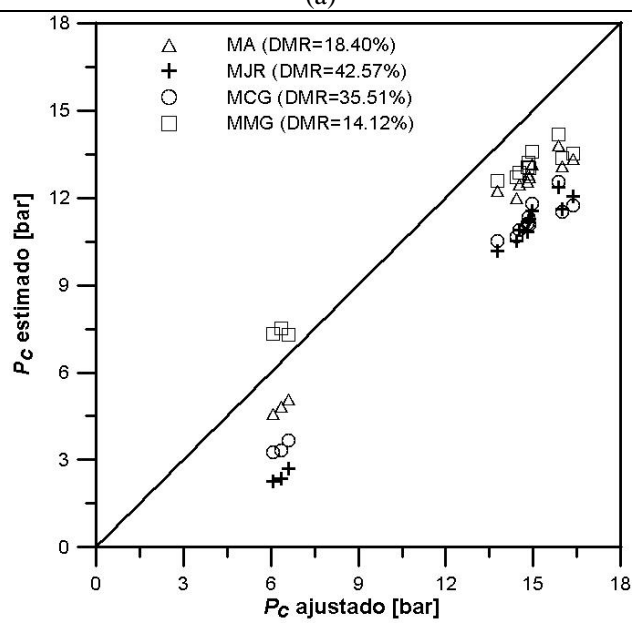

(c)

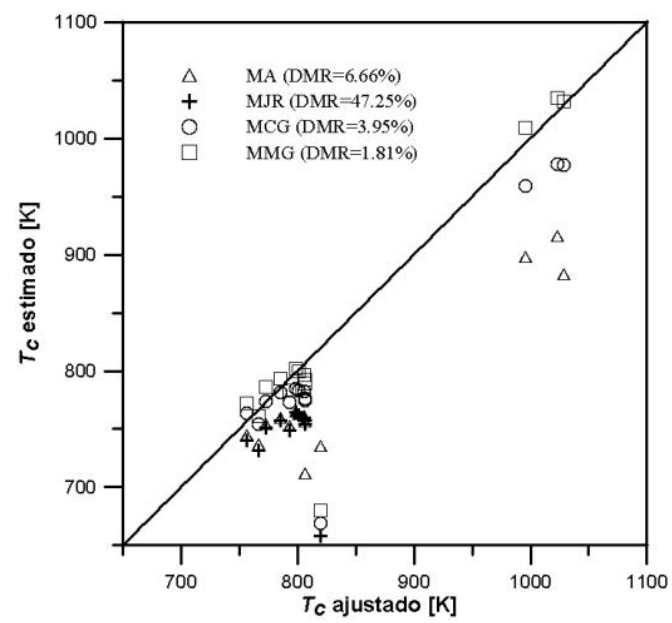

(b)

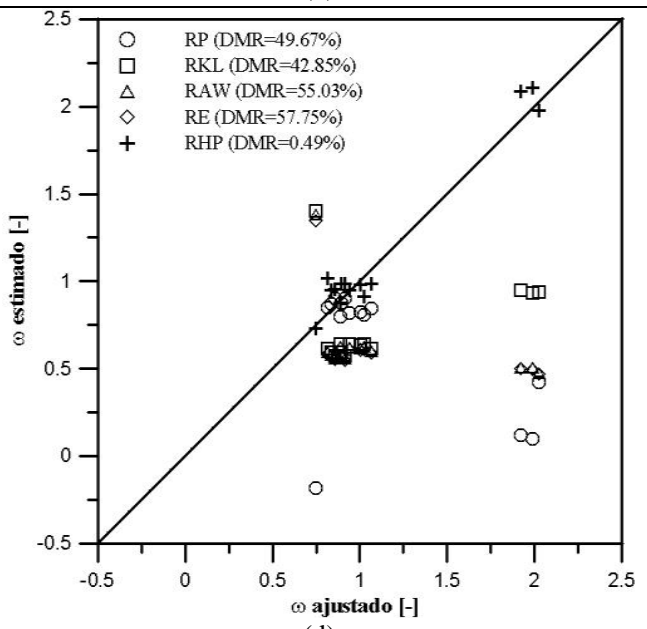

(d)

Figura 2 - (a)Visão qualitativa dos ajustes de temperatura normal de ebulição para triglicerídeos e ésteres. Dados experimentais vide: Graboski e McCormick, 1998; Perry e Green, 1999; Goodrum e Geller, 2002, Santander et al., 2012. (b), (c) e (d) 
A Tabela 3 mostra os resultados obtidos para avaliação da capacidade calorífica de líquidos utilizando a equação de Bondi, tendo sido a capacidade calorífica de gases estimada pelos MJR e MRD. Nesta Tabela, ao lado dos desvios médios relativos são computados os desvios mínimo e máximo obtidos, $N_{p}$ representa o número de dados experimentais. Nota-se nesta Tabela que os métodos MJR e MRD são próximos quanto à estimativa da capacidade calorífica de líquidos. O MJR foi selecionado para cálculos posteriores.

Tabela 3 - Desvios obtidos em para os métodos de estimação de capacidade calorífica de gases Morad et al. (2000), Bommel et al. ( 2004), DIPPR

\begin{tabular}{|c|c|c|c|c|}
\hline \multirow{2}{*}{ Substância } & \multicolumn{2}{|c|}{$\begin{array}{c}\text { Dados } \\
\text { experimentais }\end{array}$} & \multicolumn{2}{|c|}{ DMR [\%] } \\
\hline & $\begin{array}{l}\text { Intervalo } \\
\text { deT }[K]\end{array}$ & $\mathbf{N}_{\mathbf{p}}$ & MJR & MRD \\
\hline Tripalmitina & $353-453$ & 6 & $0,49(0,08-0,91)$ & $0,42(0,07-0,80)$ \\
\hline Tristearina & $353-453$ & 6 & $1,24(0,52-2,45)$ & $0,79(0,05-1,63)$ \\
\hline Trioleína & $333-453$ & 7 & $7,29(12,62-2,04)$ & $1,19(0,01-2,26)$ \\
\hline Metil Palmitato & $303-353$ & 6 & $2,40(2,15-2,51)$ & $3,09(2,98-3,16)$ \\
\hline Metil Estearato & $320-350$ & 4 & $2,27(2,16-2,37)$ & $3,19(2,93-3,34)$ \\
\hline Glicerol & $293-513$ & 12 & $9,77(1,12-22,43)$ & $12,85(1,22-36,10)$ \\
\hline
\end{tabular}

O ajuste qualitativo dos dados de entalpia de vaporização e de entalpia de formação das substâncias à 298 K são apresentadas na Figura 5. O cálculo de entalpia de vaporização foi realizado com o MCG. Para as substâncias que se encontravam sólidas nesta temperatura (TP, TE, MP, ME e EE) foram obtidos dados de entalpia de fusão na $T$ de referência.

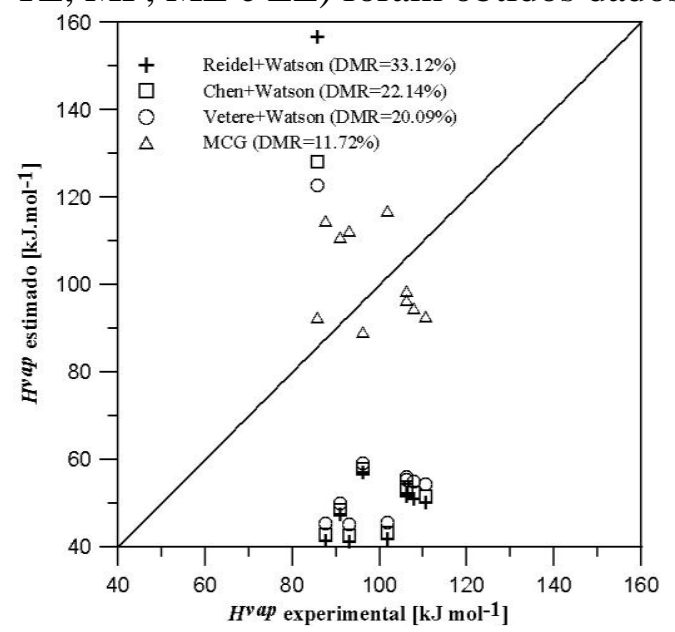

(a)

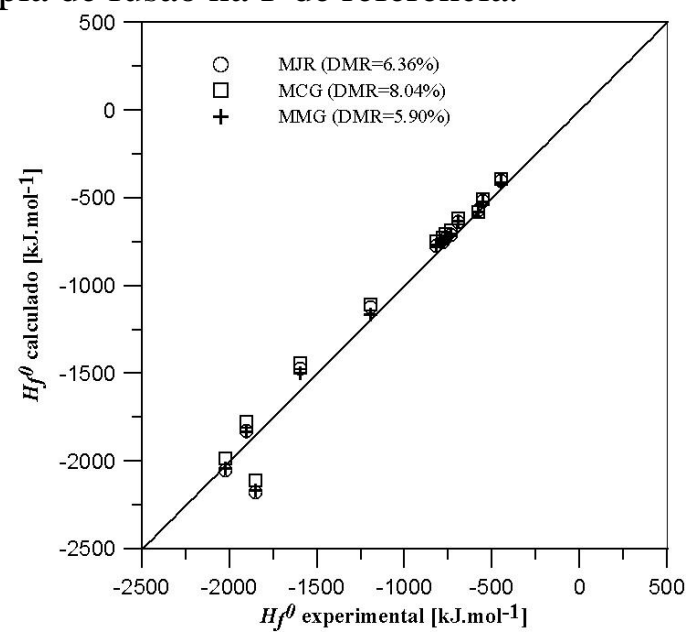

(b)

Figura 5 - Avaliação qualitativa de entalpias de vaporização (a) e de formação do gás ideal no estado padrão (b) (Referências: Bastos et al.,1988; Freedman e Bagby, 1989; Lipkind et al., 2007; Silva et al., 2011; DIPPR).

Trata-se de um dado pouco investigado para estas substâncias a energia livre de Gibbs de formação no estado padrão do gás ideal. Os valores experimentais obtidos e respectivos desvios são apresentados na Tabela 4. Devido à escassez de dados e não uniformidade de desvios obtida 
para as substâncias em questão, não foi possível inferir sobre o melhor método. Para cálculos posteriores, foi adotado o MJR.

Tabela 4 - Valores experimentais e desvios obtidos para cada método

\begin{tabular}{cccccc}
\hline \multirow{2}{*}{ Substância } & $\Delta G_{f(g a s)}^{0}$ & \multirow{2}{*}{ Referência } & \multicolumn{3}{c}{ DMR (\%) } \\
\cline { 4 - 6 } & {$[\mathbf{k J} / \mathbf{m o l}]$} & MJR & MCG & MMG \\
\hline Etil Estearato & $-136,2$ & Bucalá et al., 2005 & 17,30 & 29,50 & 15,27 \\
Metil Oleato & $-117,00$ & Bucalà et al., 2005 & 3,46 & 10,90 & 5,52 \\
Glicerol & $-424,37$ & Entropia absoluta à 298 K de & 3,33 & 7,05 & 36,27 \\
\hline
\end{tabular}

\subsection{Confecção de curvas do local geométrico do ponto crítico}

As "curvas críticas" para diversas misturas óleos vegetais+metanol e +etanol obtidas são apresentadas na Figura 6. O primeiro ponto à esquerda representa o ponto crítico de álcool puro, enquanto que o último ponto à direta representa óleo puro. Os óleos selecionados, ricos em ácidos graxos insaturados, apresentam ponto crítico praticamente idênticos. As curvas apresentadas servem como guia no planejamento de experimentos com alcoóis supercríticos. As curvas críticas apresentam ponto de máxima pressão em torno de 3-5\% de triglicerídeo. Quanto maior a cadeia o álcool menor a pressão crítica da mistura reacional.

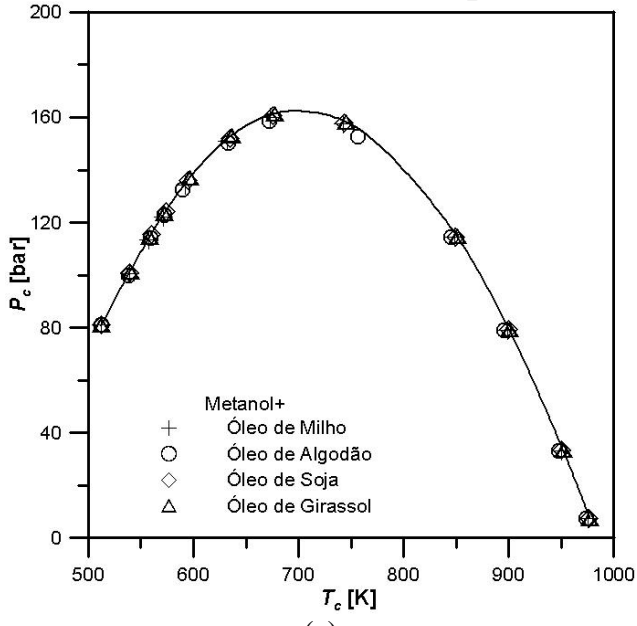

(a)

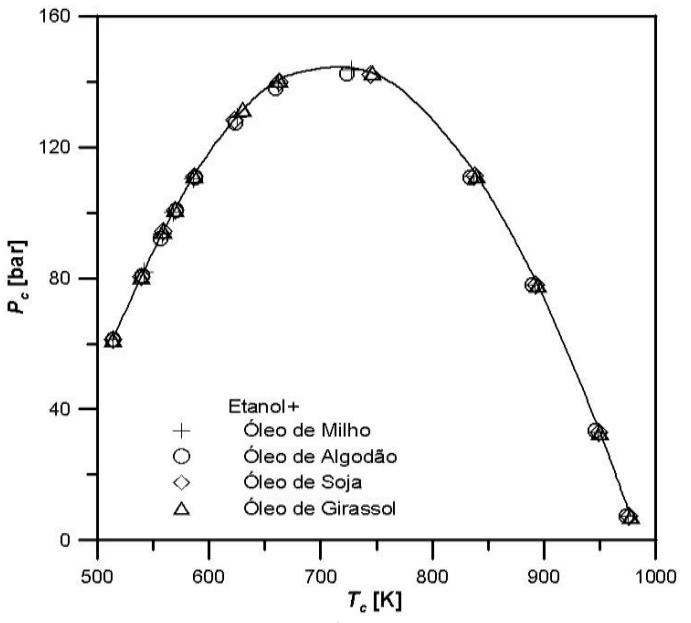

(b)

Figura 6 - Curvas críticas para os sistemas pseudobinários óleo-metanol (a) e óleo-etanol (b). Razões molares de álcool/óleo de: 0, 1, 3, 5 10, 15, 20, 30, 40, 50, 90 e 100.

\subsection{Cálculo do Equilíbrio Químico}

Para um mesmo álcool, a variação da entalpia e energia livre de Gibbs das reações à 298 $\mathrm{K}$ são iguais, independente do óleo transesterificado pois os mesmos grupos funcionais se mantêm à cada etapa da reação. Além disso, a utilização de métodos contribuição de grupos para os cálculos não diferencia as constantes de equilíbrio das três etapas reacionais (pois o mesmo grupo funcional é transferido em cada etapa reacional), sendo assim, para cada temperatura 
avaliada tem-se a mesma constante de equilíbrio para as três reações avaliadas. A Tabela 5 mostra as entalpias, energias livre de Gibbs das reações à 298 K e logaritmo natural da constante de equilíbrio no intervalo de temperatura de 300 à $700 \mathrm{~K}$.

Tabela 5 - Entalpias e energias livre de Gibbs da reação à $298 \mathrm{~K}$ e range da constante de equilíbrio

$\begin{array}{cccc}\text { Álcool } & \Delta \mathrm{H}_{\mathrm{R}}(298 \mathrm{~K})[\mathrm{J} / \mathrm{mol}] & \Delta \mathrm{G}_{\mathrm{R}}(298 \mathrm{~K})[\mathrm{J} / \mathrm{mol}] & \text { Range } \ln \left(\mathrm{K}_{\mathrm{eq}}\right) \\ \text { Metanol } & -14630 & -17320 & 6,95-3,47 \\ \text { Etanol } & -1019 & -2370 & 0,95-0,47\end{array}$

Durante os cálculos de composição de equilíbrio químico calculou-se conversão completa para todos os dois alcoóis mantendo-se o estado supercrítico da mistura reacional. Sendo que para uma mesma temperatura e pressão, as maiores conversões são primeiro para metanol e depois etanol. Alguns cálculos de rendimento em ésteres são apresentados a seguir na Tabela 6. Nesta Tabela, para o metanol, a pressão está fixa em 160 bar e temperaturas variando de $512 \mathrm{~K}$ à $700 \mathrm{~K}$ e razões molares variando de 3:1 à 40:1. Como esperado, o excesso de álcool desloca o equilíbrio para a direita, favorecendo a produção de ésteres. Quanto maior a temperatura, há uma tendência para o decréscimo do rendimento da reação para as menores razões molares. Os resultados para o etanol são semelhantes ao metanol, caindo o rendimento em ésteres em torno de $2 \%$ para a pressão de 140 bar.

Como não há variação do número de mols, o efeito das pressões pode ser analisado pela variação da fugacidade dos componentes em mistura variando o grau de avanço das reações. Para as razões molares de 20:1 à 40:1 de álcool:triglicerídeos variando as pressões de operação da condição de crítica da mistura até 300 bar a variação do rendimento em ésteres foi desprezível, indicando que se o estado supercrítico da mistura é alcançado o aumento da pressão não afeta o equilíbrio. As conclusões obtidas neste trabalho são próximas das obtidas por Anikeev et al. (2012).

Tabela 6 - Rendimento em ésteres avaliando o efeito da temperatura e razão molar metanol:óleo de soja

\begin{tabular}{cccccc}
\hline $\mathrm{T}[\mathrm{K}]$ & \multicolumn{5}{c}{ Razões molares } \\
\cline { 2 - 6 } & $3: 1$ & $10: 1$ & $20: 1$ & $30: 1$ & $40: 1$ \\
\hline 512 & 98 & 98 & 100 & 100 & 100 \\
550 & 98 & 97,89 & 99,89 & 100 & 100 \\
600 & 97,11 & 97,45 & 99,45 & 100 & 100 \\
650 & 96,33 & 97,00 & 99,00 & 100 & 100 \\
700 & 95,39 & 96,51 & 98,51 & 100 & 100
\end{tabular}

\section{CONCLUSÕES}

- É possível, a partir de dados experimentais disponíveis na literatura validar propriedades de mono, di, triglicerídeos, e ésteres etílicos e metílicos estimadas por métodos de contribuição de grupos e outras relações termodinâmicas simples. - Quando à construção de curvas críticas das misturas de alcoóis de cadeia curta e triglicerídeos pode-se notar que existe um ponto de máxima pressão, característico de misturas não ideais. - Os cálculos de equilíbrio 
químico mostram que independente do excesso de álcool a conversão dos triglicerídeos é completa e pode-se obter rendimentos próximos dos máximos para razões molares à partir de 20:1, altas pressões de trabalho que sejam próximas ao máximo pressão crítica da mistura reacional e altas temperaturas próximas ao ponto crítico do álcool puro.

\section{AGRADECIMENTOS}

Os autores agradecem à FAPEMIG (Processo PCE-00089-14) pelo apoio concedido. Ao CNPQ e à Vale pelo apoio financeiro.

\section{REFERÊNCIAS}

ANIKEEV, V.; STEPANOV, D.; YERMAKOVA, A. Thermodynamics of phase and chem. equilibrium in the proc. of biodiesel fuel synthesis in sub and supercritical methanol. Ind. Eng. Chem. Res., v. 51, p. 4783-4796, 2012.

ANISTECU, G.; DESHPANDE, A.; TAVLARIDES, L. L. Integrated Technology for Supercritical Biodiesel Production and Power Cogeneration. Energy Fuels, v. 22, p. 1391-1399, 2008.

BASTOS, M. et al. Thermodynamic properties of glycerol enthalpies of combustion and vaporization and the heat capacity at 298 K. J. Chem. Thermodynamics, v. 20, p. 1353-1359, 1988.

BOMMEL, M. J.; OONK, H. A. J.; MILTENBURG, J. C. Heat capacity of the m. esters of n-carboxylic acids from methyl octanoate to methyl eicosanoate between 5 K and 350 K. J. Chem. Eng. Data, v. 49, p. 1036-1042, 2004.

BUCALÁ, V. et al. Analysis of solvent-free ethyl oleate enzymatic synthesis at equilibrium conditions. Enzyme Microb. Technol., v. 38, p. 914-920, 2006.

DIPPR. Disponível eletronicamente em: http://dippr.byu.edu/students/. Acesso em janeiro de 2013.

FREEDMAN, B.; BABGY, M. O. Heats of combustion of fatty esters and triglycerides. J. Am. Oil Chem. Soc., v. 66, p. 1601-1605, 1989.

GOODRUM, J. W.; GELLER, D. P. Rapid thermogravimetric measurements of boiling points and vapor pressure of saturated long-chain triglycerides. Bioresour. Technol., v. 84, p. 75-80, 2002.

GRABOSKI, M. S.; MCCORMICK, R. L. Combustion of fat and vegetable oil derived fuels in diesel engines. Prog. Energy Combust. Sci., v. 24, p. 125-164, 1998.

HAN, B. X.; PENG, D. Y. A group-contribuition correlation for predicting the acentric factors of organic compounds. Can. J. Chem. Eng., v. 71, p. 332-334, 1993.

KESLER, M.G., LEE, B.I., Improve Predictions of Enthalpy of Fractions. Hydroc. Process., v. 30, p. 153-158, 1976. LIPKIND,D.; KAPUSTIN, Y.; UMNAHANANT, P.; CHICKOS, J. S. The vaporization and vapor pressure of a series of unsaturated fatty acid m. esters by correlation. Thermochim. Acta, v. 456, p. 94-101, 2007.

MA, F.; HANNA, M. A. Biodiesel production: a review. Bioresour. Technol., v. 70, p. 1-15, 1999.

MARRERO, J.; GANI, R. Group-contribution based estimation. Fluid Phase Equilib., v. 183-184, p. 183-208, 2001.

MORAD, N. A.; KAMAL, A. A. M.; PANAU, F.; YEW, T. W. Liquid specific heat capacity estimation for fatty acids, triacylglycerols, and veg. oils based on their fatty acid composition. J. Am. Oil Chem. Soc., v. 77, n. 9, 2000.

PERRY, H. R.; GREEN, D. W. Perry's Chemical engineers' handbook. 7th edition, 1999.

PFOHL, O.; PETKOV, S.; BRUNNER, G. PE 2000: A powerful tool to correlate phase equilibria. 1 ed. Herbert Utz Verlag, Munchen, 2000. $151 \mathrm{p}$.

POLING, B. E; PRAUSNITZ, J. M.; O'CONNELL, J. P. The Properties of Gases and Liquids. 5 ed. McGraw-Hill, New Yord, 2001. 803 p.

RIHANI, D. N.; DORAISWAMY, L. K. Estimation of heat capacity of organic compounds from group contributions. Ind. Eng. Chem. Fundam., v. 4, p. 17-21, 1965.

SANTANDER, C. M. G. et al. Measurements of normal boiling points of fatty acid ethyl esters and triacylglycerols by thermogravimetric analysis. Fuel, v. 92, p. 158-161, 2012.

STEPANOV, D. A.; ERMAKOVA, A.; ANIKEEV, V. I. Calculations of phase equilibria for mixtures of triglycerides, fatty Acids and their esters in lower alcohols. Russ. J. Phys. Chem. A, v. 85, p. 21-25, 2011. 